\title{
Evaluation of cervical posture improvement of children with cerebral palsy after physical therapy based on head movements and serious games
}

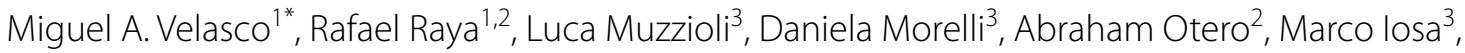 \\ Febo Cincotti $^{3}$ and Eduardo Rocon ${ }^{1}$
}

From 4th International Work-Conference on Bioinformatics and Biomedical Engineering-IWBBIO 2016 Grenada, Spain. 20-22 April 2016

\section{${ }^{*}$ Correspondence:}

miguel.velasco@csic.es

${ }^{1}$ Neural and Cognitive

Engineering Group, Centre

for Automation and Robotics

(CAR) CSIC-UPM, Ctra. Campo

Real Km 0.2, 28500 Arganda

del Rey, Spain

Full list of author information

is available at the end of the article

\begin{abstract}
Background: This paper presents the preliminary results of a novel rehabilitation therapy for cervical and trunk control of children with cerebral palsy (CP) based on serious videogames and physical exercise.

Materials: The therapy is based on the use of the ENLAZA Interface, a head mouse based on inertial technology that will be used to control a set of serious videogames with movements of the head.

Methods: Ten users with CP participated in the study. Whereas the control group $(n=5)$ followed traditional therapies, the experimental group $(n=5)$ complemented these therapies with a series of ten sessions of gaming with ENLAZA to exercise cervical flexion-extensions, rotations and inclinations in a controlled, engaging environment.
\end{abstract}

Results: The ten work sessions yielded improvements in head and trunk control that were higher in the experimental group for Visual Analogue Scale, Goal Attainment Scaling and Trunk Control Measurement Scale (TCMS). Significant differences (27\% vs. $2 \%$ of percentage improvement) were found between the experimental and control groups for TCMS $(p<0.05)$. The kinematic assessment shows that there were some improvements in the active and the passive range of motion. However, no significant differences were found pre- and post-intervention.

Conclusions: Physical therapy that combines serious games with traditional rehabilitation could allow children with CP to achieve larger function improvements in the trunk and cervical regions. However, given the limited scope of this trial $(n=10)$ additional studies are needed to corroborate this hypothesis.

Keywords: Cerebral palsy, Cervical posture, Inertial sensors, Serious games provided you give appropriate credit to the original author(s) and the source, provide a link to the Creative Commons license, and indicate if changes were made. The Creative Commons Public Domain Dedication waiver (http://creativecommons.org/publicdomain/zero/1.0/) applies to the data made available in this article, unless otherwise stated. 


\section{Background}

Cerebral palsy (CP) is a disorder of posture and movement due to a defect or lesion in the immature brain [1]. It is the most common cause of permanent physical impairment in childhood and the prospect of survival in these individuals has increased in recent years [2]. CP affects between 2 and 3 per 1000 live-births [3], reported for the European registers by the Surveillance of Cerebral Palsy European Network (SCPE), which also presented a consensus on the definition [4], classification, and description of CP [5]. $\mathrm{CP}$ is often associated to sensory deficits, cognition impairments, communication and motor disabilities, behavior issues, seizure disorder, pain, and secondary musculoskeletal problems.

People with CP frequently show low performance in activities of the daily living (ADL) due to their limited limb, trunk, and head control. Unfortunately, the poor head and trunk control in CP produce limitations beyond function. An effective oral functioning for feeding begins with attaining better head stability to improve jaw control [6]. In individuals with cervical hypotonia (as shown in Fig. 1) the limitations can be so severe that the infant may experience difficulty swallowing and breathing.

\section{Assessment of cerebral palsy}

When assessing people with $\mathrm{CP}$, many factors and symptoms need to be monitored. The functional consequences of different health states are of special interest. The Gross Motor Function Classification System (GMFCS) has been widely employed internationally to group individuals with CP into one of five levels based on functional mobility or activity limitation [7]. So has the Manual Ability Classification System (MACS) [8], which describes manipulation skills. However, the assessment and conclusions in mild cases may vary by the subjective examinations of various professionals. Therefore, a combination of significant motor developmental delay and abnormalities in the neurologic examination is required to make a diagnosis. A promising approach is the use of normal and abnormal general movement patterns [9].

In the medical field there is a need for small ambulatory sensor systems for measuring the kinematics of body segments. Motion sensing, by means of inertial sensors, can provide a real breakthrough in this field [10]. As a result, inertial sensors have been chosen for different applications focused on people with CP. Examples of this are the evaluation of clinical spasticity [11], the quantification of standing balance by assessing

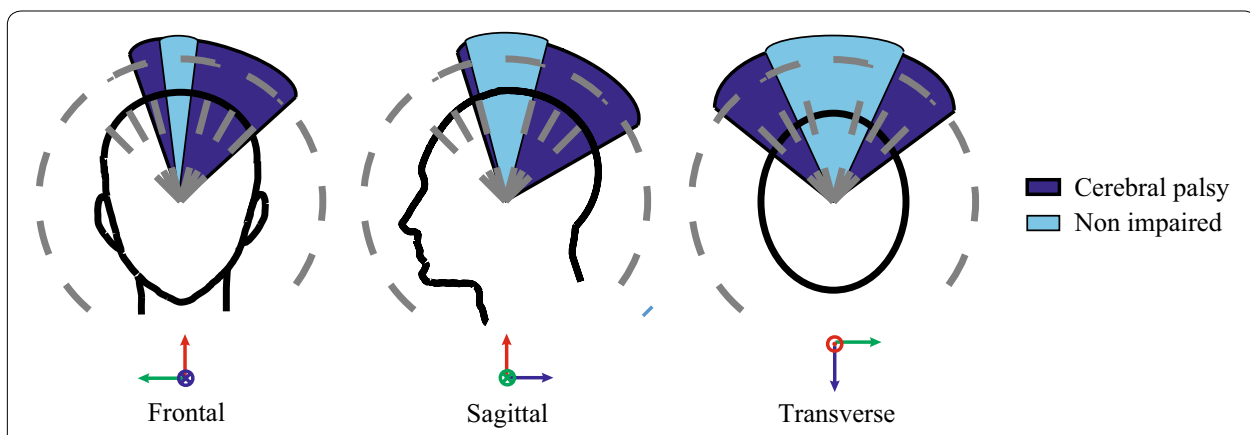

Fig. 1 Angular orientations in frontal, sagittal and transverse planes measured in two people using a head mouse: an individual with hypotonic CP (dark blue) and a non-impaired user (light blue) 
displacement of the center of mass [12], and the assessment of the predominant motor signs [13].

\section{Therapies and treatments}

The treatments for people with CP range from physical therapy to medication and surgery. Traditionally, they follow two basic principles [14]: emphasis on the normalization of the quality of movement; and emphasis on functional activities, which focuses on the development of skills necessary for the ADLs [15].

The priorities in the management of $\mathrm{CP}$ are currently changing. Whereas traditional strategies focused on promoting compensation, novel early treatments aim at restoring motor function. The reason behind this is the increasing knowledge about neuroplasticity, i.e. the ability of the neurons to reorganize their structure and function after an injury. The reorganization occurs in response to different factors, including physical training [16]. The task-oriented therapies aim to improve the movement and the posture of the patient by the repetitive training of a certain functional task. In children with $\mathrm{CP}$, the research focuses on the assessment and treatment of the upper and lower extremities to improve their performance in ADLs. In contrast, the literature on the trunk and head control is scarce [17]. The physiotherapy for the promotion of postural control and balance consists of postural orientation exercises, and exercises to strengthen the neck, back, and the musculature of the upper limb. It also includes exercises to improve the relationship between the visual environment and an internal frame of reference [18]. This strategy can be very effective in the short-term. However, in long-term rehabilitation programs, the patient often loses motivation after repeating the very same exercises weekly. Once a patient starts losing focus on the program, the therapy loses effectiveness [19].

Some experiments such as those conducted by Kramer et al. [20], Lancioni et al. [21] and Shih et al. proved that the use of biofeedback training during several weeks yields improvements in head control which last up to 16 weeks once the training is discontinued. In those experiments, the biofeedback was provided by various systems: a head position trainer (HPT), a set of micro-switches, and the Nintendo Wii Balance Board [22].

\section{A new therapy based on videogames for physical rehabilitation}

In this paper, we present a proof of concept of a rehabilitation therapy for the enhancement of head and trunk posture in children with CP. It is based on active head exercises performed through serious videogames controlled with ENLAZA, an interface based on inertial technology. We aim to develop evidence-based criteria for the integration of these exercises into the traditional therapies. Moreover, we intend to determine their role in maximizing head control in children with CP. We hypothesize that the users can improve their head posture by using the ENLAZA interface due to the neuroplasticity.

\section{Materials}

\section{The ENLAZA interface}

Raya et al. proposed the ENLAZA interface, an adapted input device for users with severe motor disorders (especially CP) who cannot use traditional solutions such 
as mice, joysticks or trackballs to access the computer [23]. The ENLAZA interface (depicted in Fig. 2) allows the users to control the cursor of the computer with movements of their heads. It consists of a head-set with a cap and an inertial measurement unit, IMU, (Werium S.L., Spain). ENLAZA integrates a tridimensional (3D) accelerometer, a 3D gyroscope, and a 3D magnetometer to measure 3D acceleration (caused by motion and gravity), 3D angular velocity, and 3D earth magnetic field. Its data fusion algorithm estimates the orientation of the IMU and enables the measurement of changes in inclination smaller than $1.0^{\circ}$ and $1-2^{\circ}$ of heading accuracy.

For the purpose of this study, the mouse pointer implements an absolute mapping, meaning that there is a one-to-one correspondence between head orientation and location of the pointer. After a calibration process, all pixels in the screen are reachable for the user's cervical range of motion. During the calibration, a therapist adjusts the gain of the transfer function which translates the orientation of the head into a location of the pointer on the screen. A Robust Kalman Filter (RKF) was developed to facilitate fine motor control based on the characterization of involuntary movements found in users with CP [24].

\section{Serious games}

The usefulness of the serious game relies on being designed to follow a series of requirements indicated by physiotherapists and considering the desirable features for rehabilitation $[19,25]$ :

- Meaningful play: The relationship between the player's interactions and the responses of the system must be consistent.

- Engagement: The game must be challenging but not frustrating. By maintaining an optimal difficulty and by including motivational elements, the apparition of fatigue and boredom can be prevented.

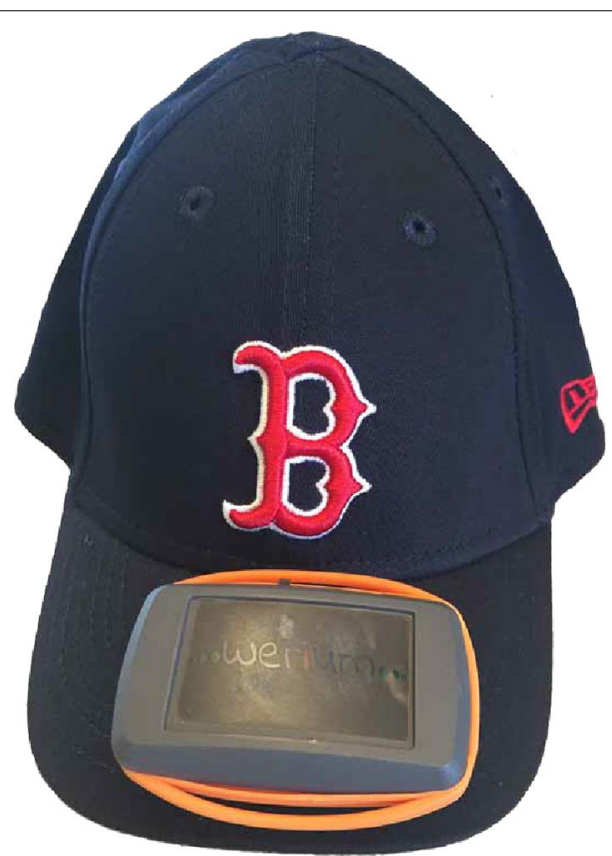

Fig. 2 The ENLAZA interface (Werium Solutions S.L., Spain) 
- In addition, the inclusion of monitoring mechanisms simplifies the therapist's work.

Six videogames were specially designed and developed in Visual C\# and the framework . NET 4.0 to be played with the ENLAZA interface. additionally, another set of six commercial off-the-shelf videogames were adapted to be played with this system. These videogames have the characteristics enumerated above:

- They are fun and systematic: there are clear objectives for the user and the task and duration are detailed before the game starts.

- All the games have different levels of difficulty and they use colors or images to represent abstract concepts as time. In addition, the games provide the participants with visual and auditory feedback.

- There is a systematic record of all the scores achieved by the participants, the orientation of the head during the game and other parameters which measure task performance. Those metrics can be examined by the therapist for further analysis.

\section{Methods}

Ten children with CP were recruited by the Fondazione Santa Lucia (FSL, Rome, Italy) to participate in this study. The inclusion/exclusion criteria can be found in Table 1. Five of the participants, the experimental group (aged $4.8 \pm 3.0$ years old), wore ENLAZA and played our selection of serious videogames. The five remaining children (aged $11.2 \pm 3.8$ years old) were grouped as controls for the experiment and followed the traditional physical and occupational therapy.

Randomization leaded to casual differences between the two groups at T0 (the beginning of the experiments). The impairment in the control group was on average more severe from a clinical point of view than that in experimental group, however these differences were not statistically significant $(\mathrm{p}=0.222$ for VAS, $\mathrm{p}=0.421$ for TCS, $\mathrm{p}=0.095$ for GMFM-88, Mann Whitney $\mathrm{u}$ test). A significant difference was found in terms of age ( $\mathrm{p}<0.05, \mathrm{t}$ test), but it was higher in control group, so it should be considered as a potential disadvantage for experimental group. Furthermore, this difference should not be considered a critical issue because within-group analysis of the improvements will be calculated.

Table 1 Inclusion/exclusion criteria for rehabilitation therapy based on head movements and serious videogames

\begin{tabular}{ll}
\hline Inclusion criteria & Exclusion criteria \\
\hline $\begin{array}{l}\text { Males and females, aged 4-21 years old } \\
\begin{array}{l}\text { Diagnosed CP and cervical hypotonia or difficulties on } \\
\text { head control }\end{array}\end{array}$ & $\begin{array}{l}\text { Aggressive or self-injure behavior } \\
\text { Involuntary movements of the head }\end{array}$ \\
$\begin{array}{l}\text { Cognitive capacity and behavior appropriate to under- } \\
\text { stand the tasks and follow simple instruction and } \\
\text { active participation in the study }\end{array}$ & $\begin{array}{l}\text { Cervical surgery within the previous } 6 \text { months } \\
\text { Signed written informed consent by parents or legal } \\
\text { guardian }\end{array}$ \\
$\begin{array}{l}\text { Medically stable } \\
\text { Inability to control the ENLAZA system during the first } \\
\text { testing session } \\
\text { Severe visual limitations }\end{array}$ \\
\hline
\end{tabular}




\section{Assessment of movement and posture}

In the following sections, we present a kinematic and functional analysis of the improvement hypothesized. ENLAZA's inertial sensor enabled the measurement of (1) the cervical range of motion (CROM) during active movements directly performed by the child and (2) the CROM during passive mobilization assisted by the therapist. The CROM consists of three movements: flexion-extension, rotation and lateral flexion.

Additionally, four measures were chosen to quantify the improvements in the posture of the head and trunk:

- Gross Motor Function Measure-88 (GMFM-88). The items 21 and 22 assess whether the children can lift and maintain their head in a vertical position with trunk support by a therapist while sitting [26].

- Visual Analogue Scale (VAS). It consists of a line of $100 \mathrm{~mm}$ separating two labels: $0=$ "No head control" and $10=$ "Perfect head control". Parents, children, and therapists were asked to put a cross on the location of the line which they thought described best the children's level of head control.

- Goal attainment scaling (GAS). It allows the therapist to program the desired improvement and to judge if the children achieved it. In this study, Goal 1 was related to head movement and Goal 2 was related to choking/swallowing.

- Trunk Control Measurement Scale (TCMS). It measures the children's ability for static sitting balance, selective movement control, and dynamic reaching. It also gives insight into the strengths and weaknesses of the children's trunk performance [17].

\section{Evaluation of task performance}

The performance of the participants in the experimental group with during their game was quantified with two parameters: success rate and throughput.

- Success rate, $S R$ (\%), measures the number of successful movements performed to reach targets which moved vertically or horizontally.

- Throughput, TP (bits/s), is a well known parameter to quantify task performance during $1 \mathrm{D}$ and $2 \mathrm{D}$ pointing tasks. It can be calculated from the movement time (MT) and the index of difficulty $(I D)$ of the task.

$T P_{\text {avg }}=\frac{I D_{\text {avg }}}{M T_{\text {avg }}}$

The Shannon formulation used by [27] represents the $I D$ as:

$I D=\log _{2}\left(\frac{A}{W}+1\right)$

where $A$ is the amplitude of the pointing movement and $W$ is the width of the target.

\section{Procedure}

At the beginning of a work session, the participants in the experimental group had to complete a routine to calibrate the interface. The procedure consisted in maintaining the head in front of the computer screen in a stable position for a few seconds. During the rest of the session, this head orientation located the cursor at the center of the screen 
during the game. The therapist would then set a value of the cursor-device gain which enabled the participant to reach all the pixels on the screen with head movements.

The protocol in a work session of the experimental group was the following:

1. Measurement of the passive CROM.

2. Measurement of the active CROM.

3. Serious games controlled with ENLAZA.

- Practice time with free controlled off-the-shelf videogames.

- Reaching tasks with targets that moved vertically (falling targets) to exercise rotations.

- Reaching tasks with targets that moved horizontally to exercise the flexion-extension.

- 2D pointing tasks. There were four series of four tasks of increasing difficulty, $I D_{i}=\{1.32,1.8,2.0,2.58$ bits $\}$. In this case, all the targets were static.

All the participants were sitting in front of a 17 in. computer screen, at a distance of $50 \mathrm{~cm}$ approximately as illustrated in Fig. 3 . The screen resolution was $136 \times 768$ pixels. The whole session lasted about 25-30 min.

\section{Statistical analysis}

The mean and standard deviation were used for the description of clinical scale scores. The percentage improvement was evaluated as

$$
\text { Improvement }=\frac{\text { Post-value }- \text { Pre-value }}{\text { Pre-value }} \cdot 100
$$

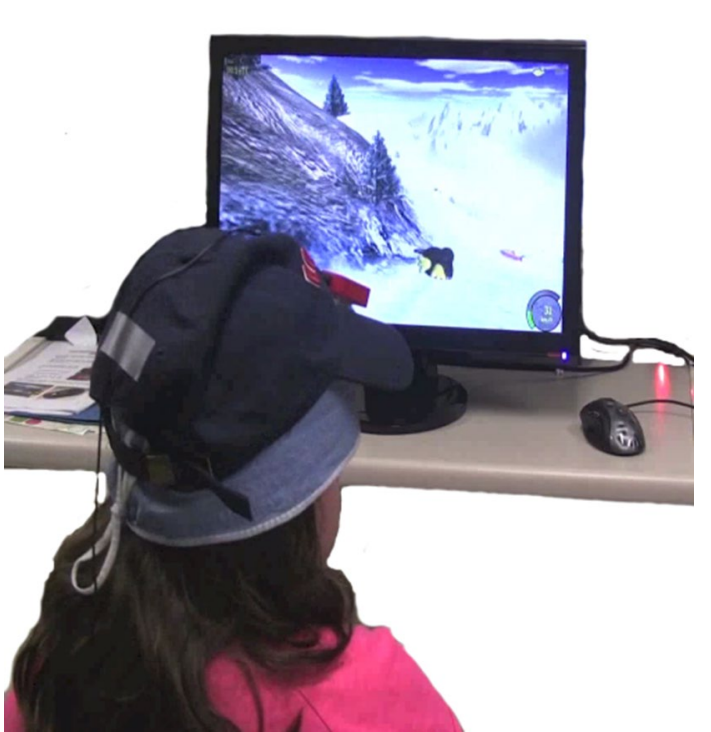

Fig. 3 A training session with ENLAZA. The user has a wired IMU attached to a baseball cap and plays the game Extreme Tux Racer 
The non-parametric Wilcoxon signed ranks (WSR) test was used for within group analysis in order to compare clinical scores at T0 (the beginning of the experiments) and after the last work-session (T1), $\alpha=0.05$, whereas the Mann-Whitney u test was used for the between group comparisons at the T0 and T1, separately $(\alpha=0.05)$.

\section{Results}

\section{Functional assessment}

Table 2 depicts the mean clinical scores pre- and post-intervention in the experimental group. The changes in the experimental group were statistically significant in terms of head control, visuomotor control assessed by GAS-score and TCMS ( $<<0.05)$. The gross motor functioning improved slightly but the value of $p$ did not achieve the statistical significant threshold. The items 21 and 22 of GMFM- 88 remained unaltered in the experimental and control groups.

In the control group (Table 3) statistically significant improvements occurred in terms of head control and visuomotor control $(\mathrm{p}<0.05)$, but in terms of trunk control or gross motor functioning. It implied that the percentage of improvement of trunk control was significantly higher in the experimental group with respect to the control group (about $+27 \%$ vs. $+2 \%$, respectively, as reported in Table 4 ). The other percentage changes, despite being higher in the experimental group, were not statistically different between the two groups, see Fig. 4.

\section{Kinematic assessment}

The ranges of motion measured before and after the therapy showed a rise of active ROM in all three movements. The percentages of increment were $+20,+38$ and $+85 \%$, achieving $93^{\circ}, 90^{\circ}$ and $145^{\circ}$ for active flexion-extension, lateral flexion and rotation, respectively. However the improvements were not statistically significant.

The passive ROM, presented smaller changes: an increment of +5 and $+57 \%$, achieving $77^{\circ}$ and $140^{\circ}$ of passive lateral flexion and rotation. Passive range of motion during flexion experienced a small reduction $(-6 \%)$ and decremented from $86^{\circ}$ to $81^{\circ}$. No statistical significance was found in these changes.

Figure 5 illustrates the evolution of active and passive flexion-extension, rotations, and inclinations of one of the participants in the experimental group.

\section{Task performance}

The participants in the control group experienced an improvement in task performance after ten work sessions, but the differences were not significant. The mean value of

Table 2 Clinical scores for experimental group (p value refers to Wilcoxon signed rank test)

\begin{tabular}{lccc}
\hline Experimental group & Pre & Post & Sig. \\
\hline VAS & $6.4 \pm 1.1$ & $7.6 \pm 1.3$ & $* \uparrow$ \\
GAS & $22.8 \pm 0.4$ & $64.3 \pm 3.6$ & $* \uparrow$ \\
TCMS & $19.4 \pm 47.5$ & $24.2 \pm 17.9$ & $* \uparrow$ \\
GMFM-88 & $44.4 \pm 0.20$ & $50.2 \pm 27.8$ & N.s. \\
\hline
\end{tabular}

Significance: ${ }^{*}<0.05$ 
Table 3 Clinical scores for control group (p value refers to Wilcoxon signed rank test)

\begin{tabular}{lccc}
\hline Control group & \multicolumn{1}{c}{ Pre } & Post & Sig. \\
\hline VAS & $5.4 \pm 1.1$ & $6.2 \pm 1.3$ & $* \uparrow$ \\
GAS & $24.8 \pm 1.2$ & $63.3 \pm 5.9$ & $* \uparrow$ \\
TCMS & $9.6 \pm 13.2$ & $10.0 \pm 13.7$ & N.s. \\
GMFM-88 & $23.0 \pm 13.3$ & $23.3 \pm 13.6$ & N.s.
\end{tabular}

Significance: ${ }^{*}<0.05$

Table 4 Percentage improvements in clinical scores for experimental, vs. control group (p value refers to Mann Whitney u test)

\begin{tabular}{lccc}
\hline Scale & Experimental & Control group & Sig. \\
\hline VAS & $18.9 \pm 6.0 \%$ & $15.2 \pm 9.4 \%$ & N.s. \\
GAS & $181.3 \pm 17.0 \%$ & $155.6 \pm 27.9 \%$ & N.s. \\
TCMS & $27.2 \pm 11.5 \%$ & $1.8 \pm 4.1 \%$ & $* \downarrow$ \\
GMFM-88 & $11.5 \pm 18.7 \%$ & $0.8 \pm 1.77 \%$ & N.s. \\
\hline
\end{tabular}

Significance: ${ }^{*}<0.05$

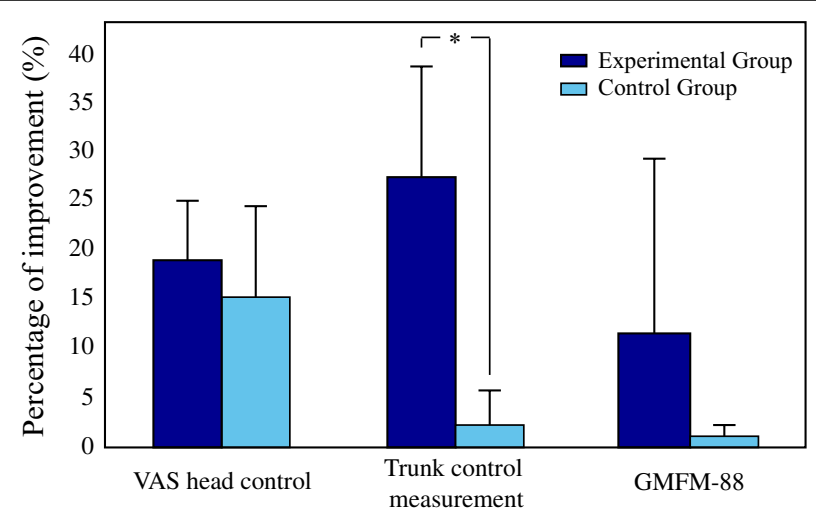

Fig. 4 Mean and standard deviations of percentage improvements in the experimental group (dark blue) and the control group (light blue)

success rate augmented from 56.8 to $65.1 \%$, whereas the mean value of throughput also rose from 0.244 to 0.482 bits/s. These values are shown in Fig. 6.

\section{Discussion}

This study aimed to quantify the improvements in head posture in five children with $\mathrm{CP}$ after ten work sessions with serious videogames controlled with a head mouse and movements of their heads. This novel therapy was followed in parallel with traditional rehabilitation therapies. We recruited a control group which followed the traditional therapies only, in order to compare the improvements in both groups.

There were improvements in four metrics (VAM, GAS, TCMS, and GMFM-88) in the experimental group, although they were not significant in the case of GMFM-88. The improvements were generally larger for the experimental group and significant differences were found in TCMS between the groups. The percentage of improvement in 

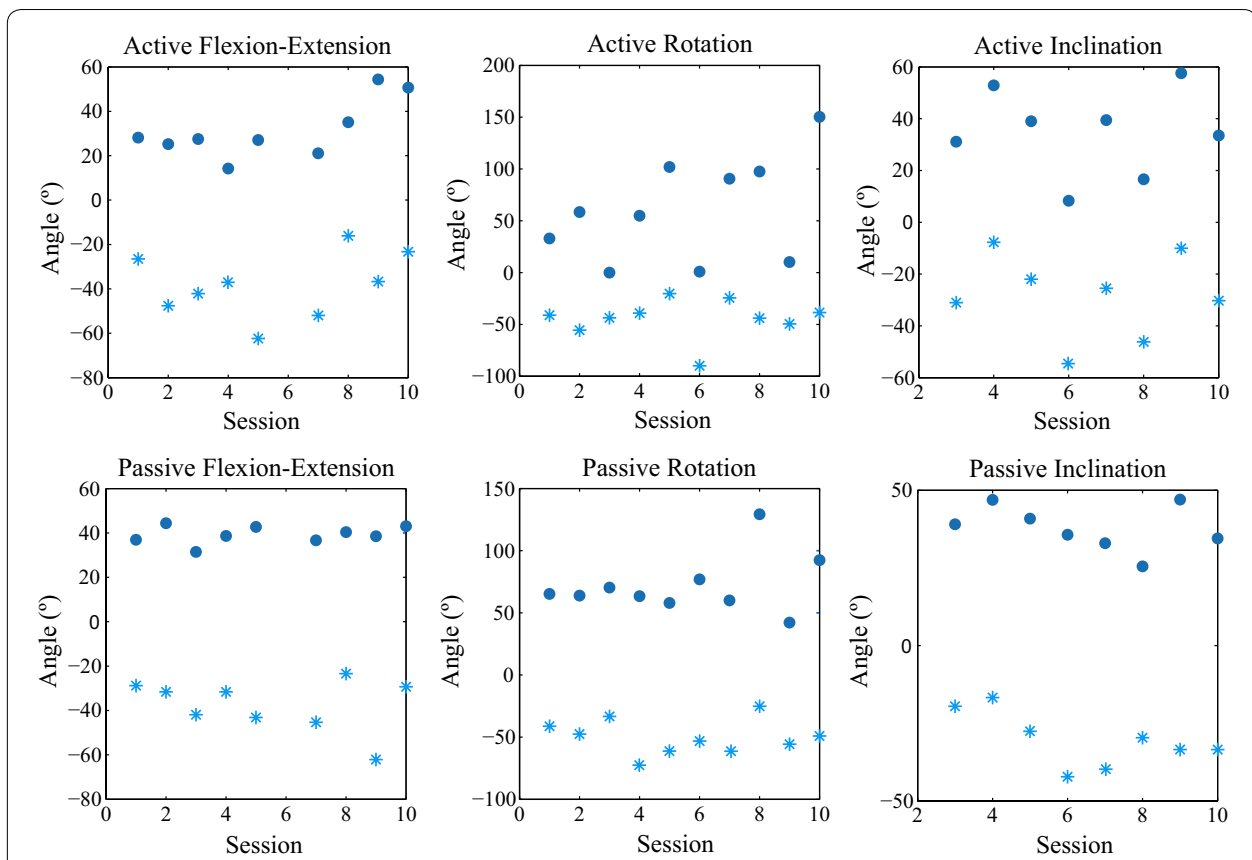

Fig. 5 Evolution of the ROMs measured for the participant CP1.The circle represents the maximum angles measured; the star the minimum angles recorded

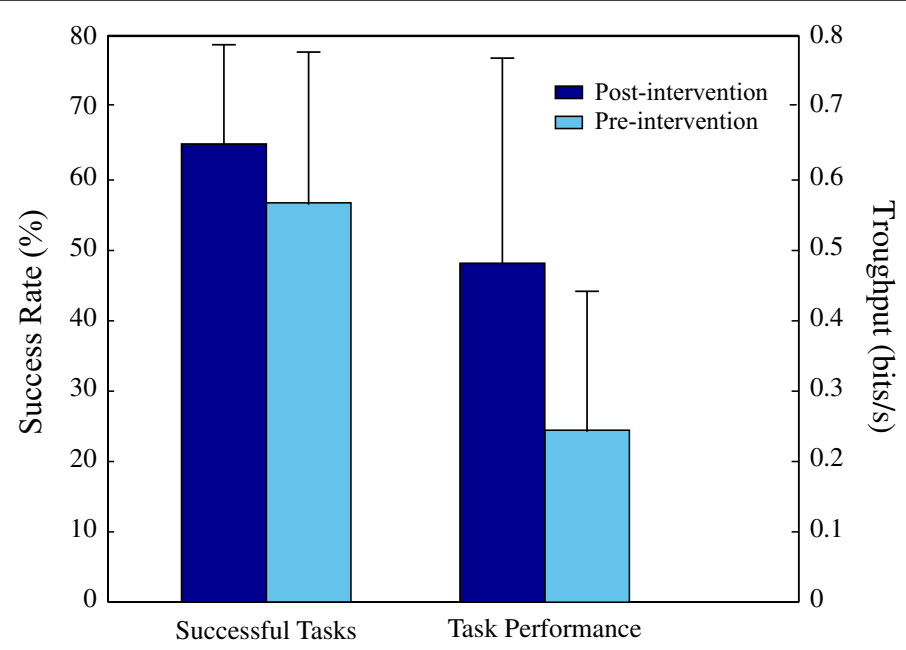

Fig. 6 Mean values of task performance (success rate and throughput) pre- and post-intervention

trunk control is indeed remarkable and shows the potential of this kind biofeedback in rehabilitation therapies.

Despite the lack of statistical significance in the improvements, the values active and passive ROM for flexion, lateral-flexion and extension after ten work sessions are closer to the physiologically normal ROMs, i.e. $55^{\circ}, 90^{\circ}$ and $120^{\circ}$ for flexion-extension, inclination, and rotation.

We found some limitations during our experiments which could have influenced our measurements and should be addressed in future trials. To begin with, the motor 
disorder was more severe in the control group (although not significantly). In addition, a sample size of ten participants (including five controls) could be considered to be too small to provide statistical strength, given the great heterogeneity of the motor disorders of our participants. In addition, the randomization used group the participants led to two groups quite different. However, no significant difference was detected in terms of disability level, and that in age was potentially in favor of the control group. Last, we must bear in mind the fact that we should update the videogames periodically in order to keep our patients motivated.

There are some technical aspects, which also influenced the measurement of the CROM. For instance, the accuracy of the IMU was high enough to enable a comfortable control of the videogames. However, it was far from the one that could have been reached with a motion capture system based on optical markers. Similarly, using only one IMU to estimate the CROM was problematic because any movement in the trunk reflected in the orientation of the head and affected the measurement. The therapists approached this issue by providing the participants with the poorest trunk postural control with pelvic and torso support. Nonetheless, two IMUs located in the head (C0) and torso (T4) are required for a better measurement of the CROM. With this approach CROM could be estimated with the rotation of the IMU located in $\mathrm{C} 0$ with respect to the one located in T4.

Unfortunately, we did not performed any analysis of the usability in this study. Nevertheless, the results of previous experiments show that ENLAZA meets some of the criteria stated by ISO 9241-Part 9. "Requirements for non-keyboard input devices", such as the adherence to Fitts's law [24]. In addition to this, all the children enrolled in the experimental group, even those of very young age, performed all the planned sessions of rehabilitation. This confirmed the compliance of children in using this device, suggesting its usability in CP. Furthermore, despite it was out of our purposes and due only to randomization process, testing the device on very young children suggest its usability also in older children. Further studies should assess the satisfaction of children, parents and therapists in using this device.

In future experiments, we will use two IMUs to improve the measurement of the CROM. We also intend to recruit a larger group in a multi-center study in order to look for greater significance in the functional and kinematic evaluation. Moreover, we are currently working on the development of a rehabilitation platform with our videogames and other games controlled by movements of the trunk or the upper limbs. Our results suggest that this kind of platform could be a powerful tool for the rehabilitation of $\mathrm{CP}$.

\section{Conclusion}

In this work, we proved that a therapy for the rehabilitation of head and trunk motor control with inertial sensors and serious games as a complement to traditional therapies is possible. Furthermore, we found that the improvements due to this novel therapy are larger than those achieved with traditional therapies alone. Despite our modest sample, the experiments yielded very promising results. Future rehabilitation treatments in physical and occupational therapy will promote the motivation of the patients and be more effective by including the use of adapted serious videogames. 


\section{Declarations}

Authors' contributions

$M A V, R R, E R$ and $M I$ designed the study. MAV and RR developed the set of serious games and processed the data from the inertial sensors and the videogames. They lead the writing process. LMD, MMI and FC recruited the participants in Italy and designed the control and experimental groups. They were in charge of supervising the experiments and calculating the clinical scores. All the authors wrote and reviewed the manuscript. All authors read and approved the final manuscript.

\section{Author details}

1 Neural and Cognitive Engineering Group, Centre for Automation and Robotics (CAR) CSIC-UPM, Ctra. Campo Real Km 0.2, 28500 Arganda del Rey, Spain. ${ }^{2}$ Department of Information Technologies, Universidad CEU San Pablo, Urbanización Montepríncipe, 28668 Boadilla del Monte, Spain. ${ }^{3}$ Fondazione Santa Lucia, FSL, Via Ardeatina, 306, 00142 Rome, Italy.

\section{Acknowledgements}

Authors would like to thank the members and staff in FSL.

\section{Competing interests}

The authors declare that they have no competing interests.

\section{Availability of data and materials}

Data sharing is not applicable to this article as no datasets were generated during the current study.

\section{Ethics approval and consent to participate}

The experiments had received approval by the Ethics Committee of FSL. A parent or guardian of all the children signed an informed consent prior to the beginning of the study.

\section{Funding}

The research work and publication costs were funded by NetMD (RTC-2015-3967-1), NeuroMOD (DPI2015-68664-C41-R), and InterAAC (RTC-2015-4327-1). All these projects were financed by the Spanish Ministry of Economy, Industry and Competitiveness.

\section{About this supplement}

This article has been published as part of BioMedical Engineering OnLine Volume 16 Supplement 1, 2017: Selected articles from the 4th International Work-Conference on Bioinformatics and Biomedical Engineering-IWBBIO 2016. The full contents of the supplement are available online at https://biomedical-engineering-online.biomedcentral.com/articles/ supplements/volume-16-supplement-1.

\section{Publisher's Note}

Springer Nature remains neutral with regard to jurisdictional claims in published maps and institutional affiliations.

Published: 18 August 2017

\section{References}

1. Bax M, Goldstein M, Rosenbaum P, Leviton A, Paneth N, Dan B, Jacobsson B, Damiano D. Proposed definition and classification of cerebral palsy, April 2005. Dev Med Child Neurol. 2005;47(8):571-6. doi:10.1017/ S001216220500112X.

2. Blair E. Epidemiology of the cerebral palsies. Orthop Clin N Am. 2010;41(4):441-55. doi:10.1016/j.ocl.2010.06.004

3. Johnson A, Perinatal N, Unit E, Road O. Prevalence and characteristics of children with cerebral palsy in Europe. Dev Med Child Neurol. 2002:44(9):633-40.

4. Cans C. Surveillance of cerebral palsy in Europe: a collaboration of cerebral palsy surveys and registers. Dev Med Child Neurol. 2007:42(12):816-24. doi:10.1111/j.1469-8749.2000.tb00695.x.

5. Krägeloh-Mann I, Cans C. Cerebral palsy update. Brain Dev. 2009;31 (7):537-44. doi:10.1016/j.braindev.2009.03.009.

6. Redstone F, West JF. The importance of postural control for feeding. Pediatr Nurs. 2004;30(2):97-100.

7. Palisano RR, Rosenbaum P, Bartlett D, Livingstone M. Gross motor function classification system —expanded and revised. Child A Glob J Child Res. 2008;50(10):744-50.

8. Eliasson A-C, Krumlinde-Sundholm L, Rösblad B, Beckung E, Arner M, Ohrvall A-M, Rosenbaum P. The Manual Ability Classification System (MACS) for children with cerebral palsy: scale development and evidence of validity and reliability. Dev Med Child Neurol. 2006;48(7):549-54. doi:10.1017/S0012162206001162.

9. Van der Heide J, Paolicelli PB, Boldrini A, Cioni G. Kinematic and qualitative analysis of lower-extremity movements in preterm infants with brain lesions. Phys Ther. 1999;79(6):546-57.

10. Kim M, Kim BH, Jo S. Quantitative evaluation of a low-cost noninvasive hybrid interface based on EEG and eye movement. IEEE Trans Neural Syst Rehabil Eng. 2015;23(2):159-68. doi:10.1109/TNSRE.2014.2365834.

11. Cutti AG, Cappello A, Davalli A. A new technique for compensating the soft tissue artefact at the upper-arm: in vitro validation. J Mech Med Biol. 2005;5(2):1-15. doi:10.1142/S0219519405001485.

12. Ghasemzadeh H, Jafari R, Prabhakaran B. A body sensor network with electromyogram and inertial sensors: multimodal interpretation of muscular activities. IEEE Trans Inf Technol Biomed. 2010;14(2):198-206. doi:10.1109/ TITB.2009.2035050. 
13. Velasco MA, Raya R, Ceres R, Clemotte A, Ruiz Bedia A, Gonzalez Franco T, Rocon E. Positive and negative motor signs of head motion in cerebral palsy: assessment of impairment and task performance. IEEE Syst J. 2016;10(3):967-73. doi:10.1109/JSYST.2014.2318075.

14. Bower E. The multiply handicapped child. In: Wilson BA, et al., McLellan LD, Editors. Rehabilitation studies handbook. New York: Cambridge University Press; 1997. p. 315-54.

15. Ketelaar M, Vermeer A, Hart $H$, van Petegem-van Beek E, Helders PJ. Effects of a functional therapy program on motor abilities of children with cerebral palsy. Phys Ther. 2001;81(9):1534-45.

16. Aisen ML, Kerkovich D, Mast J, Mulroy S, Wren TAL, Kay RM, Rethlefsen SA. Cerebral palsy: clinical care and neurological rehabilitation. Lancet Neurol. 2011;10(9):844-52. doi:10.1016/S1474-4422(11)70176-4.

17. Heyrman L, Molenaers G, Desloovere K, Verheyden G, De Cat J, Monbaliu E, Feys H. A clinical tool to measure trunk control in children with cerebral palsy: The Trunk Control Measurement Scale. Res Dev Disabil. 2011;32(6):2624-35. doi:10.1016/j.ridd.2011.06.012.

18. Ledebt A, Becher J, Kapper J, Rozendaalr RM, Bakker R, Leenders IC, Savelsbergh GJP. Balance training with visual feedback in children with hemiplegic cerebral palsy: effect on stance and gait. Mot Control. 2005;9(4):459-68.

19. Jaume-I-Capó A, Martinez-Bueso P, Moya-Alcover B, Varona J. Interactive rehabilitation system for improvement of balance therapies in people with cerebral palsy. IEEE Trans Neural Syst Rehabil Eng. 2014;22(2):419-27. doi:10.1109/ TNSRE.2013.2279155.

20. Kramer JF, Ashton B, Brander R. Training of head control in the sitting and semi-prone positions. Child Care Health Dev. 1992;18(6):365-76.

21. Lancioni GE, Singh NN, O'Reilly MF, Sigafoos J, Didden R, Oliva D, Severini L. Fostering adaptive responses and head control in students with multiple disabilities through a microswitch-based program: follow-up assessment and program revision. Res Dev Disabil. 2007;28(2):187-96. doi:10.1016/j.ridd.2006.02.005.

22. Shih $\mathrm{CH}$, Shih $\mathrm{CT}$, Chu CL. Assisting people with multiple disabilities actively correct abnormal standing posture with a Nintendo Wii Balance Board through controlling environmental stimulation. Res Dev Disabil. 2010;31(4):936-42. doi:10.1016/j.ridd.2010.03.004.

23. Raya R, Roa JO, Rocon E, Ceres R, Pons JL. Wearable inertial mouse for children with physical and cognitive impairments. Sens Actuators A Phys. 2010;162(2):248-59. doi:10.1016/j.sna.2010.04.019.

24. Raya R, Rocon E, Gallego JA, Ceres R, Pons JL. A robust Kalman algorithm to facilitate human-computer interaction for people with cerebral palsy, using a new interface based on inertial sensors. Sensors. 2012;12(3):3049-67. doi:10.3390/s120303049.

25. Burke JW, McNeill MDJ, Charles DK, Morrow PJ, Crosbie JH, McDonough SM. Optimising engagement for stroke rehabilitation using serious games. Vis Comput. 2009;25(12):1085-99. doi:10.1007/s00371-009-0387-4.

26. Weis R. Gross motor function measure (GMFM-66 and GMFM-88) user's manual. Eur J Paediatric Neurol. 2004;8(2):111-2. doi:10.1016/j.ejpn.2003.11.003.

27. MacKenzie IS. A note on the information-theoretic basis of Fitts'law. J Mot Behav. 1989;21 (3):323-30. doi:10.1080/00 222895.1989.10735486.

Submit your next manuscript to BioMed Central and we will help you at every step:

- We accept pre-submission inquiries

- Our selector tool helps you to find the most relevant journal

- We provide round the clock customer support

- Convenient online submission

- Thorough peer review

- Inclusion in PubMed and all major indexing services

- Maximum visibility for your research

Submit your manuscript at www.biomedcentral.com/submit 